\title{
A PROOF OF VAN DOUWEN'S RIGHT IDEAL THEOREM
}

\author{
DENNIS E. DAVENPORT AND NEIL HINDMAN
}

(Communicated by Dennis Burke)

\begin{abstract}
In 1979 Eric K. van Douwen announced a powerful theorem about the Stone-Čech compactification of a discrete semigroup which he called The Right Ideal Theorem. Its proof, however, was lost with his untimely death. In this paper we present a proof of the theorem and a derivation of some of its corollaries.
\end{abstract}

\section{INTRODUCTION}

In a letter to the second author dated 22 February 1979, Eric K. van Douwen announced several results including his Right Ideal Theorem and some of its corollaries. After van Douwen's death in 1987, the second author prepared The Čech-Stone compactification of a discrete groupoid [2] for publication and was able, with assistance, to prove most of the results in that letter. A proof of the featured result of the letter, however, was elusive. We present such a proof here.

Given a discrete semigroup $(S, \cdot)$ it is well known that the operation extends to the Stone-Čech compactification $\beta S$ of $S$ in such a way that for each $p \in$ $\beta S$, the function $\lambda_{p}: \beta S \rightarrow \beta S$ defined by $\lambda_{\rho}(q)=p \cdot q$ is continuous, and for each $x \in S$, the function $\rho_{x}: \beta S \rightarrow \beta S$ defined by $\rho_{x}(p)=p \cdot x$ is continuous. (See [2] or [4] for an elementary derivation of these facts.) Moreover if $S$ is cancellative then the space $U(S)$ of uniform ultrafilters on $S$ is a subsemigroup of $\beta S$. (Much weaker hypotheses suffice-see [3, Theorem 2.5].)

Sections 2 and 3 of this paper present preliminary lemmas for the Right Ideal Theorem. The featured theorem is presented in $\S 4$.

4.1. Theorem (The Right Ideal Theorem). If $(S, \cdot)$ is a cancellative semigroup with $|S|=\lambda \geq \omega$, then there exists a decomposition $\mathscr{I}$ of $U(S)$ into pairwise disjoint subsets such that

(1) $|\mathscr{I}|=2^{2^{\lambda}}$,

Received by the editors February 5, 1990.

1980 Mathematics Subject Classification (1985 Revision). Primary 22A30; Secondary 54O35, $22 \mathrm{~A} 15$.

Key words and phrases. Right Ideal Theorem, left-topological semigroup.

The second author gratefully acknowledges the support of the National Science Foundation, Grant DMS-8901058. 
(2) for each $I \in \mathscr{I}, I$ is nowhere dense in $U(S)$,

(3) for each $I \in \mathcal{F}, I$ is a right ideal of $\beta S$, and

(4) for all $I \in \mathscr{I}$ and all $p \in I, \operatorname{cl}(S \cdot p) \subseteq I$.

(The cardinality of $\mathscr{I}$ was stated erroneously in [2] because of an error by the second author.)

Throughout this paper we will let $(S, \cdot)$ be a discrete cancellative semigroup with $|S|=\lambda \geq \omega$. We take the points of $\beta S$ to be the ultrafilters on $S$, identifying the points of $S$ with the principal ultrafilters. Given $A \subseteq S, \bar{A}=$ $\{p \in \beta S: A \in p\}$. While $\bar{A}=\mathrm{cl}_{\beta S} A$, the point of more interest is that $\{\bar{A}: A \subseteq S\}$ forms a basis for the open sets of $\beta S$. The operation - on $\beta S$ can be characterized as follows. Given $A \subseteq S$, and $p, q \in \beta S, A \in p \cdot q$ if and only if $\{x: A / x \in p\} \in q$ where $A / x=\{y \in S: y \cdot x \in A\}$.

Recall that if $p$ is an ultrafilter on $S$, then the norm of $p$, denoted by $\|p\|$, is given by $\|p\|=\min \{|A|: A \in p\}$. Also, $p$ is a uniform ultrafilter, that is, $p \in U(S)$, if and only if $\|p\|=|S|=\lambda$. Thus $p$ is a uniform ultrafilter if and only if for each $A \in p,|A|=\lambda$.

Our set theoretic notation is standard. In particular, cardinals are ordinals and $\omega$ is the first infinite cardinal.

\section{A Relation on $U(S)$}

Since we want a decomposition of $U(S)$, one approach is to define an equivalence relation on $U(S)$. We are guided in this by the conclusions of the theorem. We are specifically given that for $I \in \mathscr{I}$ and $p \in I, \operatorname{cl}(S \cdot p) \subseteq I$. Further, for $I \in \mathscr{I}$ and $p \in I$ we have $p \cdot \beta S \subseteq I$ and $p \cdot \beta S=\lambda_{p}[\operatorname{cl} S]=\operatorname{cl} \lambda_{p}[S]=\operatorname{cl}(p \cdot S)$. Thus if $p, q \in U(S)$ and $(\operatorname{cl}(S \cdot p) \cup \operatorname{cl}(p \cdot S)) \cap(\operatorname{cl}(S \cdot q) \cup \operatorname{cl}(q \cdot S)) \neq \varnothing$ we must have that $p$ and $q$ are equivalent.

2.1. Definition. $R=\{(p, q) \in U(S) \times U(S):(\operatorname{cl}(S \cdot p) \cup \operatorname{cl}(p \cdot S)) \cap(\operatorname{cl}(S \cdot q) \cup$ $\operatorname{cl}(q \cdot S)) \neq \varnothing\}$.

It is clear that $R$ is both symmetric and reflexive. However, $R$ need not be transitive. Recall that given two relations $C$ and $D$, the composition $C \circ D$ is defined by $(x, y) \in C \circ D$ if and only if there exists $z$ with $(x, z) \in D$ and $(z, y) \in C$. Further we write $C^{2}$ for $C \circ C$ and so on.

2.2. Definition. Let $\sim$ be the transitive closure of $R$. That is, for $p, q \in$ $U(S), p \sim q$ if and only if there is some $n \in \mathbf{N}$ with $p R^{n} q$.

One gets immediately that $\sim$ is an equivalence relation on $U(S)$. We denote the $\sim$-equivalence class of $p$ by $[p]$.

2.3. Lemma. Let $p \in U(S)$. Then $[p]$ is a right ideal of $\beta S$ and for each $q \in[p], \operatorname{cl}(S \cdot q) \subseteq[p]$.

Proof. We first show that $[p]$ is a right ideal of $\beta S$. Let $q \in[p]$ and let $r \in \beta S$. We first observe that $q \cdot r \in U(S)$. Indeed let $A \in q \cdot r$. Then 
$\{x \in S: A / x \in q\} \in r$ so pick $x \in S$ with $A / x \in q$. Then $|A / x|=\lambda$, and because $S$ is right cancellative, $\rho_{x}$ takes $A / x$ one-to-one into $A$. Now $q \cdot r \cdot r \in q \cdot \beta S \cap(q \cdot r) \cdot \beta S$ so $(q \cdot r) R q$ so $q \cdot r \sim q$ and hence $q \cdot r \in[p]$.

To see that $\operatorname{cl}(S \cdot q) \subseteq[p]$, let $r \in \operatorname{cl}(S \cdot q)$. We claim that $\operatorname{cl}(S \cdot r) \cap \operatorname{cl}(S \cdot q) \neq \varnothing$ (so that $r \sim q$ and hence $r \sim p$ ). In fact we claim $\operatorname{cl}(S \cdot r) \subseteq \operatorname{cl}(S \cdot q)$. To see this let $v \in \operatorname{cl}(S \cdot r)$ and let $A \in v$. We show $\bar{A} \cap(S \cdot q) \neq \varnothing$. Since $A \in v$ and $v \in \operatorname{cl}(S \cdot r)$, pick $x \in S$ with $x \cdot r \in \bar{A}$. Since $\lambda_{x}$ is continuous pick $B \in r$ with $x \cdot \bar{B} \subseteq \bar{A}$. Now $r \in \operatorname{cl}(S \cdot q)$ so pick $y \in S$ with $y \cdot q \in \bar{B}$. Then $x \cdot y \cdot q \in \bar{A}$ as required.

\section{THE EQUivalenCe ClASSES ARE NOWHERE DENSE}

To show that the equivalence classes are nowhere dense we will need several lemmas.

3.1. Lemma. Let $E \in[S]^{\lambda}$. Then there exist an enumeration $\left\langle a_{\sigma}\right\rangle_{\sigma<\lambda}$ of $S$, an increasing sequence $\langle m(\sigma)\rangle_{\sigma<\lambda}$ in $\lambda$ and a nondecreasing function $f: \lambda \rightarrow \lambda$ such that,

(1) if $\sigma$ is a limit, then $m(\sigma)=\sup _{\delta<\sigma} m(\delta)$,

(2) if $\sigma<\omega$, then $|m(\sigma)|<\omega$,

(3) if $\omega \leq \sigma<\lambda$, then $|m(\sigma)|=|\sigma|$,

(4) for all $\sigma<\lambda, m(f(\sigma)) \leq \sigma<m(f(\sigma)+1)$, and

(5) for all $\sigma<\lambda, a_{m(\sigma)} \in E$.

Further, if we let $T=\left\{\left(a_{\nu}, a_{\tau}\right)\right.$ : there exist $\gamma<f(\tau), \mu<f(\nu)$, and $z \in S$ with (i) $z=a_{\tau} a_{\gamma}$ or $z=a_{\gamma} a_{\tau}$ and (ii) $z=a_{\nu} a_{\mu}$ or $\left.z=a_{\mu} a_{\nu}\right\}$ define $B_{0}(y)=\{y\}$ and $B_{n}(y)=\left\{x \in S: x T^{n} y\right\}$ for $n>0$, then

(6) if $\sigma<\lambda, \tau<m(\sigma), n<\omega, \nu<\lambda$, and $a_{\nu} \in B_{n}\left(a_{\tau}\right)$, then $\nu<$ $m(\sigma+n)$.

Proof. Let $\left\langle b_{\sigma}\right\rangle_{\sigma<\lambda}$ be any enumeration of $S$. Given $x, z \in S$, define $D(x, z)$ $=\{y \in S: z=x \cdot y$ or $z=y \cdot x\}$. Note that because $S$ is cancellative, $|D(x, z)| \leq 2$.

We construct by induction the sequences in the statement of the lemma as well as sequences $\left\langle Z_{\sigma}\right\rangle_{\sigma<\lambda}$ and $\left\langle W_{\sigma}\right\rangle_{\sigma<\lambda}$ of subsets of $S$ satisfying

(7) for $\sigma<\lambda, Z_{\sigma}=\left\{a_{\gamma} \cdot a_{\tau}: \gamma<m(\sigma)\right.$ and $\left.\tau<\sigma\right\} \cup\left\{a_{\tau} a_{\gamma}: \gamma<m(\sigma)\right.$ and $\tau<\sigma\}$

(8) for $\sigma<\lambda, W_{\sigma}=\left\{b_{\sigma}\right\} \cup \bigcup\left\{D\left(a_{\tau}, z\right): z \in Z_{\sigma}\right.$ and $\left.\tau \leq \sigma\right\}$, and

(9) for $\sigma<\lambda, W_{\sigma} \subseteq\left\{a_{\tau}: \tau<m(\sigma+1)\right\}$.

At a limit stage in the induction we only define $m(\sigma)=\sup _{\delta<\sigma} m(\delta)$ as required by (1). At a successor stage $\sigma=\delta+1$ we define $m(\sigma),\left\langle a_{\tau}\right\rangle_{m(\delta) \leq \tau<m(\sigma)}$, $Z_{\delta}$, and $W_{\delta}$. (The function $f$ is not defined until the induction is complete.) We verify (2) and (3) and the fact that $\langle m(\sigma)\rangle_{\sigma<\lambda}$ increases as we go along because these facts are needed to continue the construction. We verify the rest of the conclusions at the end. 
Let $\sigma$ be a limit ordinal and let $m(\sigma)=\sup _{\delta<\sigma} m(\delta)$. If $\sigma<\omega$, then $\sigma=0$ and $m(\sigma)=0$ so (2) holds. If $\sigma=\omega$ then $m(\sigma)=\sup _{n<\omega} m(n) \leq \sup _{n<\omega} \omega=$ $\omega$ while $m(\sigma)=\sup _{n<\omega} m(n) \geq \sup _{n<\omega} n=\omega$ so (3) holds. Assume $\sigma>\omega$. Then $m(\sigma)=\sup _{\delta<\sigma} m(\delta) \geq \sup _{\delta<\sigma} \delta=\sigma$ so $|m(\sigma)| \geq|\sigma|$. Also $|m(\sigma)| \leq$ $\sum_{\delta<\sigma}|m(\delta)|=\sum_{n<\omega}|m(n)|+\sum_{\omega \leq \delta<\sigma}|m(\delta)| \leq \sum_{n<\omega} \omega+\sum_{\omega \leq \delta<\sigma}|\delta| \leq \omega+$ $\sum_{\omega \leq \delta<\sigma}|\sigma| \leq \sum_{\delta<\sigma}|\sigma|=|\sigma|$. Thus again (3) holds. Trivially $m(\sigma)>m(\delta)$ for $\delta<\sigma$.

Now assume $\sigma=\delta+1$. By (2) or (3) we have $|m(\delta)|<\lambda$ so pick $a_{m(\delta)} \in$ $E \backslash\left\{a_{m(\tau)}: \tau<\delta\right\}$. Let $Z_{\delta}=\left\{a_{\gamma} \cdot a_{\tau}: \gamma<m(\delta)\right.$ and $\left.\tau<\delta\right\} \cup\left\{a_{\tau} \cdot a_{\gamma}: \gamma<m(\delta)\right.$ and $\tau<\delta\}$ and let $W_{\delta}=\left\{b_{\delta}\right\} \cup \bigcup\left\{D\left(a_{\tau}, z\right): z \in Z_{\delta}\right.$ and $\left.\tau \leq \delta\right\}$. Note that if $\delta<\omega$ then $\left|Z_{\delta}\right|<\omega$ and hence $\left|W_{\delta}\right|<\omega$. Also if $\omega \leq \delta<\lambda$, then $\left|Z_{\delta}\right| \leq$ $2 \cdot|m(\delta)| \cdot|\delta|=|\delta|$ and $\left|W_{\delta}\right| \leq 2 \cdot\left|Z_{\delta}\right| \cdot|\delta|=|\delta|$. Let $\mu=\left|W_{\delta} \backslash\left\{a_{\tau}: \tau \leq m(\delta)\right\}\right|$, let $\left\langle c_{\tau}\right\rangle_{\tau<\mu}$ be an enumeration of $W_{\delta} \backslash\left\{a_{\tau}: \tau \leq m(\delta)\right\}$, and let $m(\sigma)$ be the ordinal sum $m(\delta)+1+\mu$. For $\tau<\mu$, let $a_{m(\delta)+1+\tau}=c_{\tau}$ and observe that conclusion (9) holds at $\delta$. If $\sigma<\omega$ we have $|m(\sigma)| \leq|m(\delta)|+1+\left|W_{\delta}\right|<\omega$. If $\omega \leq \sigma$ (so $\omega \leq \delta$ ) we have $|m(\sigma)| \geq|m(\delta)|=|\delta|=|\sigma|$ and $|m(\sigma)| \leq$ $|m(\delta)|+|\mu| \leq|\delta|+|\delta|=|\delta|=|\sigma|$. Thus hypotheses (2) and (3) hold. Also observe $m(\sigma) \geq m(\delta)+1$.

The construction is complete. It is clear from the construction that the sequence $\left\langle a_{\sigma}\right\rangle_{\sigma<\lambda}$ is one-to-one. Also, given $\delta, b_{\delta} \in W_{\delta}$ so $b_{\delta}=a_{\tau}$ for some $\tau<m(\delta+1)$. Thus $\left\langle a_{\sigma}\right\rangle_{\sigma<\lambda}$ is an enumeration of $S$. Hypotheses (1), (2), (3), and (5) follow immediately from the construction. We define $f: \lambda \rightarrow \lambda$ by $f(\sigma)=\min \{\delta: \sigma<m(\delta+1)\}$. (Since $m$ is an increasing function from $\lambda$ to $\lambda$, its range is cofinal.) Immediately $\sigma<m(f(\sigma)+1)$. Suppose $\sigma<m(f(\sigma))$. Then $f(\sigma)$ must be a limit since if $f(\sigma)=\delta+1$ one has $\sigma<m(\delta+1)$ so $f(\sigma) \leq \delta$. But then $m(f(\sigma))=\sup _{\delta<f(\sigma)} m(\delta)$ so one can pick $\delta<f(\sigma)$ with $\sigma<m(\delta)$ so $\sigma<m(\delta+1)$ so again $f(\sigma) \leq \delta$, a contradiction. Thus hypothesis (4) holds.

We conclude by proving (6) by induction on $n$. If $a_{\nu} \in B_{0}\left(a_{\tau}\right)$ then $\nu=\tau<$ $m(\sigma)$ as required. Now assume $n=1$. Let $\sigma<\lambda, \tau<m(\sigma)$, and $\nu<\lambda$ with $a_{\nu} \in B_{1}\left(a_{\tau}\right)$ be given. Then $a_{\nu} T a_{\tau}$ so pick $\gamma<f(\tau), \mu<f(\nu)$, and $z \in S$ with (i) $z=a_{\tau} a_{\gamma}$ or $z=a_{\gamma} a_{\tau}$ and (ii) $z=a_{\mu} a_{\nu}$ or $z=a_{\nu} a_{\mu}$. It suffices to show that $f(\nu) \leq \sigma$, since then $\nu<m(f(\nu)+1) \leq m(\sigma+1)$. Suppose instead that $\sigma<f(\nu)$.

Now $\sigma<f(\nu)$ and $\mu<f(\nu)$ so, if $f(\nu)$ is a limit pick $\delta<f(\nu)$ with $\sigma<\delta$ and $\mu<\delta$. If $f(\nu)$ is a successor pick $\delta$ with $f(\nu)=\delta+1$ and note that $\sigma \leq \delta$ and $\mu \leq \delta$. Now $z=a_{\tau} a_{\gamma}$ or $z=a_{\gamma} a_{\tau}$. Note that since $\tau<m(\sigma), f(\tau)<\sigma$. Since $\gamma<f(\tau)<\sigma \leq \delta$ and $\tau<m(f(\tau)+1) \leq m(\sigma) \leq m(\delta)$ we have $z \in Z_{\delta}$. Also, either $z=a_{\nu} a_{\mu}$ or $z=a_{\mu} a_{\nu}$, so $a_{\nu} \in D\left(a_{\mu}, z\right)$. Since $\mu \leq \delta$ we have $D\left(a_{\mu}, z\right) \subseteq W_{\delta}$ so $a_{\nu} \in W_{\delta}$. Thus by (9), $\nu<m(\delta+1) \leq m(f(\nu))$, a contradiction.

Finally let $n \geq 1$ and assume (6) holds at $n$. Let $\sigma<\lambda, \tau<m(\sigma)$, and $\nu<\lambda$ with $a_{\nu} \in B_{n+1}\left(a_{\tau}\right)$ be given. Then $a_{\nu} T^{n+1} a_{\tau}$. Pick $\delta<\lambda$ with $a_{\nu} T a_{\delta}$ 
and $a_{\delta} T^{n} a_{\tau}$. Then $a_{\nu} \in B_{1}\left(a_{\delta}\right)$ and $a_{\delta} \in B_{n}\left(a_{\tau}\right)$. Since $a_{\delta} \in B_{n}\left(a_{\tau}\right)$ we have $\delta<m(\sigma+n)$. Since $\delta<m(\sigma+n)$ and $a_{\nu} \in B_{1}\left(a_{\delta}\right)$ we have $\nu<m(\sigma+n+1)$ as required.

3.2. Lemma. Let $E \in[S]^{\lambda}$ and let $\left\langle a_{\sigma}\right\rangle_{\sigma<\lambda},\langle m(\sigma)\rangle_{\sigma<\lambda}, f: \lambda \rightarrow \lambda, T$, and $B_{n}(y)$ be as in Lemma 3.1. Let $p, q \in U(S)$, let $n<\omega$ and assume $p R^{n} q$. Then for each $A \in q, \bigcup_{x \in A} B_{n}(x) \in p$.

Proof. We show first that

if $r \in \operatorname{cl}(S \cdot q) \cup \operatorname{cl}(q \cdot S), A \in q$, and $D=\{z \in S$ : there

(*) exist $\tau<\lambda$ and $\gamma<f(\tau)$ with $a_{\tau} \in A$ and either $z=a_{\gamma} a_{\tau}$ or $\left.z=a_{\tau} a_{\gamma}\right\}$, then $D \in r$.

Suppose instead that $S \backslash D \in r$. Assume first $r \in \operatorname{cl}(S \cdot q)$ and pick $y \in S$ with $y \cdot q \in \overline{S \backslash D}$ and pick $\gamma<\lambda$ with $y=a_{\gamma}$. Choose $E \in q$ with $\lambda_{y}[\bar{E}] \subseteq \overline{S \backslash D}$. Note that $\{\tau: f(\tau) \leq \gamma\} \subseteq\{\tau: \tau<m(\gamma+1)\}$ so $|\{\tau: f(\tau) \leq \gamma\}| \leq|m(\gamma+1)|<$ $\lambda$. Since $q \in U(S)$, this means $\left\{a_{\tau}: f(\tau)>\gamma\right\} \in q$. Pick $\tau$ with $f(\tau)>\gamma$ and $a_{\tau} \in A \cap E$. Then since $a_{\tau} \in A, a_{\gamma} a_{\tau} \in D$ while, since $a_{\tau} \in E, a_{\gamma} \cdot a_{\tau} \in S \backslash D$, a contradiction. Likewise if $r \in \operatorname{cl}(q \cdot S)$ we get a contradiction by choosing $y$ with $q \cdot y \in \overline{S \backslash D}$ and choosing $E \in q$ with $\rho_{y}[\bar{E}] \subseteq \overline{S \backslash D}$.

Now we verify the lemma for $n=1$. Assume $A \in q$ and $p R q$ and pick $r \in(\operatorname{cl}(S \cdot p) \cup \operatorname{cl}(p \cdot S)) \cap(\operatorname{cl}(S \cdot q) \cup \operatorname{cl}(q \cdot S))$. Suppose $\bigcup_{x \in A} B_{1}(x) \notin p$ and let $H=S \backslash \bigcup_{x \in A} B_{1}(x)$. Let $D=\{z \in S:$ there exist $\tau<\lambda$ and $\gamma<f(\tau)$ with $a_{\tau} \in A$ and either $z=a_{\gamma} a_{\tau}$ or $\left.z=a_{\tau} a_{\gamma}\right\}$ and let $G=\{z \in S$ : there exist $\nu<\lambda$ and $\mu<f(\nu)$ with $a_{\nu} \in H$ and either $z=a_{\nu} a_{\mu}$ or $\left.z=a_{\mu} a_{\nu}\right\}$. By $(*)$ we have $D \in r$ and, since $H \in p$ and $r \in(\operatorname{cl}(S \cdot p) \cup \operatorname{cl}(p \cdot S)), G \in r$. Pick $z \in D \cap G$, pick $\tau<\lambda$ and $\gamma<f(\tau)$ with $a_{\tau} \in A$ and either $z=a_{\gamma} a_{\tau}$ or $z=a_{\tau} a_{\gamma}$. Also pick $\nu<\lambda$ and $\mu<f(\nu)$ with $a_{\nu} \in H$ and either $z=a_{\nu} a_{\mu}$ or $z=a_{\mu} a_{\nu}$. Then $a_{\nu} T a_{\tau}$ so $a_{\nu} \in B_{1}\left(a_{\tau}\right)$, a contradiction.

Finally we assume the lemma is true for $n$, let $A \in q$, and assume $p R^{n+1} q$. Pick $r \in U(S)$ with $p R r$ and $r R^{n} q$ and let $C=\bigcup_{x \in A} B_{n}(x)$ and let $D=$ $\bigcup_{y \in C} B_{1}(y)$. Then $C \in r$ so $D \in p$. Since $D \subseteq \bigcup_{x \in A} B_{n+1}(x)$ we have $\bigcup_{x \in A} B_{n+1}(x) \in p$ as required.

We now need slightly different approaches depending on whether $\lambda>\omega$.

3.3. Lemma. Let $E \in[S]^{\lambda}$ and let $\left\langle a_{\sigma}\right\rangle_{\sigma<\lambda},\langle m(\sigma)\rangle_{\sigma<\lambda}, f: \lambda \rightarrow \lambda$, and $B_{n}(y)$ be as in Lemma 3.1. Assume $\lambda>\omega$ and for each $\sigma<\lambda$ let $y_{\sigma}=a_{m(\omega \cdot \sigma)}$. Then for $\sigma<\nu<\lambda$ we have $\left(\bigcup_{n<\omega} B_{n}\left(y_{\sigma}\right)\right) \cap\left(\bigcup_{n<\omega} B_{n}\left(y_{\nu}\right)\right)=\varnothing$.

Proof. Observe first that for each $\sigma, \omega \cdot \sigma<\lambda$ (since $|\omega \cdot \sigma|=\max \{|\omega|,|\sigma|\}<$ $\lambda)$. Consequently $y_{\sigma}$ is well defined. Now let $\sigma<\nu<\lambda$ be given and suppose we have some $z \in\left(\bigcup_{n<\omega} B_{n}\left(y_{\sigma}\right)\right) \cap\left(\bigcup_{m<\omega} B_{m}\left(y_{\nu}\right)\right)$. Pick $n, m<\omega$ with $z \in B_{n}\left(y_{\sigma}\right)$ and $z \in B_{m}\left(y_{\nu}\right)$. Then $y_{\nu} \in B_{n+m}\left(y_{\sigma}\right)$. That is $a_{m(\omega \cdot \nu)} \in$ $B_{n+m}\left(a_{m(\omega \cdot \sigma)}\right)$. Since $m(\omega \cdot \sigma)<m(\omega \cdot \sigma+1)$ we have by Lemma 3.1(6) that $m(\omega \cdot \nu)<m(\omega \cdot \sigma+1+n+m)<m(\omega \cdot \sigma+\omega)=m(\omega \cdot(\sigma+1)) \leq m(\omega \cdot \nu)$, a contradiction. 
3.4. Lemma. Let $E \in[S]^{\lambda}$ and let $\left\langle a_{\sigma}\right\rangle_{\sigma<\lambda},\langle m(\sigma)\rangle_{\sigma<\lambda}, f: \lambda \rightarrow \lambda$, and $B_{n}(y)$ be as in Lemma 3.1. Assume $\lambda=\omega$ and for each $n<\omega$ let $y_{n}=a_{m\left(n^{2}+2 n\right)}$. If $t<n<\omega$ then

(1) if $a_{l} \in \bigcup_{k=0}^{2 n} B_{k}\left(y_{t}\right)$, then $l<m\left(n^{2}+2 n\right)$ and

(2) $\left(\bigcup_{k=0}^{n} B_{k}\left(y_{n}\right)\right) \cap\left(\bigcup_{k=0}^{t} B_{k}\left(y_{t}\right)\right)=\varnothing$.

Proof. Let $t<n<\omega$ be given. To verify (1) let $a_{l} \leq \bigcup_{k=0}^{2 n} B_{k}\left(y_{t}\right)$ and pick $k \leq 2 n$ with $a_{l} \in B_{k}\left(y_{t}\right)=B_{k}\left(a_{m\left(t^{2}+2 t\right)}\right)$. Because $m\left(t^{2}+2 t\right)<m\left(t^{2}+2 t+1\right)$ we have by Lemma 3.1(6) that $l<m\left(t^{2}+2 t+1+k\right)$. Since $t^{2}+2 t+1+k \leq$ $(n-1)^{2}+2(n-1)+1+2 n=n^{2}+2 n, l<m\left(n^{2}+2 n\right)$ as required.

To verify (2) suppose we have some $x \in\left(\bigcup_{k=0}^{n} B_{k}\left(y_{n}\right)\right) \cap\left(\bigcup_{k=0}^{t} B_{k}\left(y_{t}\right)\right)$ and pick $k \leq n$ and $l \leq t$ with $x \in B_{k}\left(y_{n}\right) \cap B_{l}\left(y_{t}\right)$. Then $a_{m\left(n^{2}+2 n\right)}=y_{n} \in B_{k+l}\left(y_{t}\right)$ so by (1), $m\left(n^{2}+2 n\right)<m\left(n^{2}+2 n\right)$, a contradiction.

We are now ready for the main result of this section.

3.5. Lemma. Let $p \in U(S)$. Then $[p]$ is nowhere dense in $U(S)$.

Proof. Suppose cl $[p]$ has nonempty interior in $U(S)$ and pick $E \in[S]^{\lambda}$ with $\bar{E} \cap U(S) \subseteq \operatorname{cl}[p]$. Choose $\left\langle a_{\sigma}\right\rangle_{\sigma<\lambda},\langle m(\sigma)\rangle_{\sigma<\lambda}, f: \lambda \rightarrow \lambda$, and $B_{n}(y)$ as guaranteed for $E$ by Lemma 3.1.

Case 1. $\lambda>\omega$. For each $\sigma<\lambda$ let $y_{\sigma}=a_{m(\omega \cdot \sigma)}$ and note that by Lemma 3.1(5), $y_{\sigma} \in E$. For each $\sigma<\lambda$ let $B_{\omega}\left(y_{\sigma}\right)=\bigcup_{n<\omega} B_{n}\left(y_{\sigma}\right)$. By Lemma 3.3, if $\sigma<\nu<\lambda$ we have $B_{\omega}\left(y_{\sigma}\right) \cap B_{\omega}\left(y_{\nu}\right)=\varnothing$. Let $C=\bigcup\left\{B_{\omega}\left(y_{\sigma}\right): \sigma<\lambda\right.$ and $\sigma$ is even $\}$ and let $D=\bigcup\left\{B_{\omega}\left(y_{\sigma}\right): \sigma<\lambda\right.$ and $\sigma$ is odd $\}$. Then $C \cap D=\varnothing$. Without loss of generality assume $C \notin p$ and let $F=\left\{y_{\sigma}: \sigma<\lambda\right.$ and $\sigma$ is even $\}$ and note that $|F|=\lambda$. Pick $q \in \bar{F} \cap U(S)$. Then $q \in \bar{E} \cap U(S)$ so $q \in \operatorname{cl}[p]$. Thus $\bar{F} \cap[p] \neq \varnothing$ so pick $r \in \bar{F} \cap[p]$. Then $F \in r$ and for some $n<\omega r R^{n} p$. Then by Lemma $3.2 \bigcup_{x \in F} B_{n}(x) \in p$. But $\bigcup_{x \in F} B_{n}(x) \subseteq C$ so $C \in p$, a contradiction.

Case 2. $\lambda=\omega$. For each $n<\omega$ let $y_{n}=a_{m\left(n^{2}+2 n\right)}$ and note that by Lemma 3.1(5), $y_{n} \in E$. Let $C=\bigcup\left\{\bigcup_{k=0}^{n} B_{k}\left(y_{n}\right): n<\omega\right.$ and $n$ is even $\}$ and let $D=\bigcup\left\{\bigcup_{k=0}^{n} B_{k}\left(y_{n}\right): n<\omega\right.$ and $n$ is odd $\}$. By Lemma 3.4 we have $C \cap D=\varnothing$ so again without loss of generality $C \notin p$. Let $F=\left\{y_{2 n}: n<\omega\right\}$ and pick $q \in \bar{F} \cap U(S)$. Then $q \in \bar{E} \cap U(S)$ so $q \in \operatorname{cl}[p]$. Thus $\bar{F} \cap[p] \neq \varnothing$ so pick $r \in \bar{F} \cap[p]$. Then $F \in r$ and for some $m<\omega r R^{m} p$. Let $G=\left\{y_{2 n}: m \leq\right.$ $2 n\}$. Then $|F \backslash G|<\omega$ so $G \in r$ so by Lemma 3.2, $\bigcup_{x \in G} B_{m}(x) \in p$. But $\bigcup_{x \in G} B_{m}(x) \subseteq C$ so $C \in p$, a contradiction.

Our final preliminary result counts the equivalence classes.

3.6. Lemma. $|\{[p]: p \in U(S)\}|=2^{2^{\lambda}}$.

Proof. Let $E=S$ and let $\left\langle y_{\sigma}\right\rangle_{\sigma<\lambda}$ be as guaranteed for $E$ by Lemma 3.3 or Lemma 3.4 and let $Y=\left\{y_{\sigma}: \sigma<\lambda\right\}$. It is well known that the number 
of uniform ultrafilters on a set of cardinality $\lambda$ is $2^{2^{\lambda}}$. (See for example [1, Corollary 7.8].) It thus suffices to show that if $p$ and $q$ are distinct members of $\bar{Y} \cap U(S)$ then $[p] \neq[q]$. Suppose instead we have such distinct $p$ and $q$ with $p \sim q$. Pick $n<\omega$ with $p R^{n} q$ and since $q \neq p$, pick $B \in q \backslash p$ so that $Y \backslash B \in p$.

Case 1. $\lambda>\omega$. Let $D=B \cap Y$. Then $D \in q$ so by Lemma 3.2, $\bigcup_{x \in D} B_{n}(x) \in p$ so $\left(\bigcup_{x \in D} B_{n}(x)\right) \cap(Y \backslash B) \neq \varnothing$. Pick $\sigma, \nu<\lambda$ with $y_{\sigma} \in D$ and $y_{\nu} \in(Y \backslash B) \cap B_{n}\left(y_{\sigma}\right)$. Then $B_{0}\left(y_{\nu}\right) \cap B_{n}\left(y_{\sigma}\right) \neq \varnothing$, contradicting Lemma 3.3.

Case 2. $\lambda=\omega$. Let $D=\left\{y_{m}: m>n\right\} \cap B$. Then since $\left\{y_{m}: m \leq n\right\} \notin q$, $D \in q$. By Lemma 3.2, $\bigcup_{x \in D} B_{n}(x) \in p$ so $\left(\bigcup_{x \in D} B_{n}(x)\right) \cap(Y \backslash B) \neq \varnothing$. Pick $m>n$ and $t$ with $y_{m} \in D$ and $y_{t} \in(Y \backslash B) \cap B_{n}\left(y_{m}\right)$. Then $B_{0}\left(y_{t}\right) \cap B_{n}\left(y_{m}\right) \neq$ $\varnothing$ contradicting Lemma 3.4.

\section{The Right IDEAL Theorem}

4.1. Theorem. Let $(S, \cdot)$ be a cancellative infinite semigroup. Then there exists a decomposition $\mathscr{I}$ of $U(S)$ such that

(1) $|\mathcal{F}|=2^{2^{|S|}}$,

(2) for each $I \in \mathscr{J}, I$ is nowhere dense,

(3) for each $I \in \mathcal{I}, I$ is a right ideal of $\beta S$, and

(4) for each $I \in \mathscr{I}$ and each $p \in I, \operatorname{cl}(S \cdot p) \subseteq I$.

Proof. Let $\mathscr{I}=\{[p]: p \in U(S)\}$. Conclusion (1) is Lemma 3.6. Conclusion (2) is Lemma 3.5. Conclusions (3) and (4) are Lemma 2.4.

The following corollaries were also included in van Douwen's letter. For the first two of these corollaries observe that if $r, p \in U(S)$ and $r \cdot p=p \cdot r$ then $\operatorname{cl}(r \cdot S) \cap \operatorname{cl}(p \cdot S) \neq \varnothing$ so that $r \sim p$.

4.2. Corollary. Let $S$ be infinite and cancellative. For all $p \in U(S)$ the centralizer of $p$ in $U(S)$ is nowhere dense in $U(S)$.

Proof. Simply observe that the centralizer of $p$ is contained in $[p]$.

We denote the center of a semigroup $S$ by $Z(S)$.

4.3. Corollary. Let $S$ be infinite and cancellative. Then $Z(U(S))=\varnothing$.

Proof. Suppose $r \in Z(U(S))$. Then for all $q \in U(S), r \sim q$ so $U(S)=[r]$ contradicting conclusion (1) of Theorem 4.1.

4.4. Corollary. Let $S$ be infinite and cancellative. Then $Z(\beta S)=Z(S)$.

Proof. To see that $Z(S) \subseteq Z(\beta S)$ let $x \in Z(S)$ and let $p \in \beta S$. Suppose $x \cdot p \neq p \cdot x$ and pick $U$ and $V$ disjoint neighborhoods of $x \cdot p$ and $p \cdot x$ respectively. Since $\lambda_{x}$ and $\rho_{x}$ are continuous pick $A, B \in p$ with $\lambda_{x}[\bar{A}] \subseteq U$ and $\rho_{x}[\bar{B}] \subseteq V$. Pick $y \in A \cap B \cap S$. Then $x \cdot y \in U$ and $x \cdot y=y \cdot x \in V$, a contradiction. 
Now suppose we have some $p \in Z(\beta S) \backslash Z(S)$. We have immediately that $p \notin S$ so $\kappa=\|p\| \geq \omega$. Choose $A \in p$ with $|A|=\kappa$ and let $H$ be the subsemigroup of $S$ generated by $A$. Then $|H|=\kappa$ and $p \in U(H)$. (The extension of the operation on $S$ to $\beta S$ extends the operation on $H$ to $U(H)$.) Because $p \in Z(\beta S), p \in Z(U(H))$, contradicting Corollary 4.3.

We close by observing that one cannot replace "cancellative" in Theorem 4.1 by either "right cancellative" or "left cancellative." If one lets $S$ be any set and defines $x \cdot y=x$ for all $x, y \in s$ then for all $p, q \in \beta S$ one has $p \cdot q=p$. Picking any $q \in U(S)$ one has $S \cdot q=S$ so $\operatorname{cl}(S \cdot q)=\beta S$. Likewise if one lets $x \cdot y=y$ for all $x, y \in S$ one gets $p \cdot q=q$ for all $p, q \in \beta S$ and hence, for $q \in U(S), q \cdot S=S$ so $q \cdot \beta S=\operatorname{cl}(q \cdot S)=\operatorname{cl}(S)=\beta S$.

\section{REFERENCES}

1. W. Comfort and S. Negrepontis, The theory of ultrafilters, Springer-Verlag, Berlin, 1978.

2. E. van Douwen, The Čech-Stone compactification of a discrete groupoid, Topology Appl. (to appear).

3. N. Hindman, The ideal structure of the space of $\kappa$-uniform ultrafilters on a discrete semigroup, Rocky Mountain J. Math. 16 (1986), 685-701.

4. __ Ultrafilters and combinatorial number theory, Number Theory Carbondale (M. Nathanson, ed.) Lecture Notes in Math., vol. 751, Springer-Verlag, 1979, pp. 119-184.

Department of Mathematics, Miami University, Oxford, Ohio 45056

Department of Mathematics, Howard University, Washington, D.C. 20059 